\title{
Optimization a structure of MEMS based PDMS ferroelectret for human body energy harvesting and sensing
}

\author{
Junjie Shi ${ }^{1}$, Zhenhua Luo $^{2}$, Dibin, Zhu $^{3}$, Steve Beeby ${ }^{1^{*}}$ \\ ${ }^{1}$ Department of Electronics and Computer Science, University of Southampton, UK \\ ${ }^{2}$ School of Water, Energy and Environment, Cranfield University, UK \\ ${ }^{3}$ Energy harvesting research Group College of Engineering, Mathematics and Physical Sciences, \\ University of Exeter, UK \\ E-mail: spb@soton.ac.uk
}

\begin{abstract}
A ferroelectret is typically a charge-storing cellular foam that demonstrates excellent piezoelectric properties making them potentially suitable for both sensing and energy harvesting applications. In this work we developed a numerical finite element analysis (FEA) model to describe ferroelectret materials and to further improve their piezoelectric properties. Using this FEA model, ferroelectret materials with rectangular and parallelogram void structure were designed and then fabricated by casting polydimethysiloxane (PDMS) in microfabricated silicon moulds. The piezoelectric properties and energy harvesting output of the fabricated PDMS ferroelectrets were both simulated and evaluated experimentally. For a single layer PDMS parallelogram void structure, the predicted piezoelectric coefficient $d_{33}$ from the ANSYS simulations is around $320 \mathrm{pC} / \mathrm{N}$. The fabricated PDMS ferroelectret has a low Young's modulus of $670 \mathrm{kPa}$ and a piezoelectric coefficient of 240 $\mathrm{pC} / \mathrm{N}$. A maximum $d_{33}$ of $520 \mathrm{pC} / \mathrm{N}$ was observed in a multilayer ferroelectret structure. When applying compressive forces simulating a footstep, the material demonstrated an output power of $2.73 \mu \mathrm{W}$ when connected to a $65 \mathrm{M} \Omega$ resistive load.
\end{abstract}

\section{Keywords}

PDMS ferroelectret, MEMS fabrication, finite element method, energy harvesting

\section{Introduction}

Piezoelectric materials can be used in the direct mode as sensors or as energy harvesting materials to convert mechanical energy into electrical energy and also as actuators that converts electrical energy into mechanical energy [1-3]. Applications in energy harvesting include converting energy from human movement making piezoelectric materials a potential power source for wearable devices and electronic textiles (e-textiles). In the past, research has focused on the conventional piezoelectric materials such as lead zirconate titanate (PZT), PZT based materials, polyvinylidene fluoride (PVDF) and P(VDF-TrFE) copolymer [4-7]. PZT possesses excellent dielectric and piezoelectric properties, but is a rigid ceramic with a very high Young's modulus (63 GPa), making it unsuitable for wearable applications [8]. In contrast, PVDF is a soft polymer with low Young's modulus (2.9 GPa), but its piezoelectric charge coefficient $d_{33}$ is an order of magnitude 
lower than of PZT (20 pC/N) [9]. In addition, the energy harvesting capability is not solely determined by $d_{33}$, it also effected by permittivity of the material. The low permittivity result in high figure of merit $\left(d_{33} * g_{33}\right)$ can enhanced the energy harvesting capability. Therefore, there is a requirement to develop a compliant piezoelectric material with high levels of piezoelectric activity, low young's modulus and low permittivity suitable for wearable applications.

Ferroelectrets are materials (typically a cellular polymer foam) that store charge across its internal voids and that exhibits piezoelectric properties [8]. The typical internal structure of a ferroelectret foam is randomly arranged cellular voids with positive and negative charges trapped on opposite on each surfaces of the void. A macroscopic dipoles moment is formed by the separated charges resulting in piezoelectric-like property of ferroelectret. Due to the low elastic modulus of the ferroelectret material, the electrically charged voids undergo large deformation when compressed, the dipole moment of the ferroelectret is reduced; and the electrical filed in the internal voids is compensated by net charges which are generated on the electrodes on the outer surfaces of the ferroelectret material. A commercial polypropylene (PP) foam ferroelectret has a $d_{33}$ of 250-400 $\mathrm{pC} / \mathrm{N}$, which is comparable to the $d_{33}$ of bulk ceramic PZT $(300-650 \mathrm{pC} / \mathrm{N})$. The piezoelectric properties of a ferroelectret are dependent on the structure of the cellular voids, the material characteristics and the amount of charge trapped on the void surfaces [10-12].

Most ferroelectrets foams are produced using a blow and extrusion process on polymer films [1315]. The individual void geometry and overall cellular structure of the ferroelectret foam are typically ill-controlled due to the stochastic nature of these fabrication processes. It is anticipated that the piezoelectric properties of a ferroelectret material can potentially be improved by optimising its internal structure. A number of previous works have attempted to control the geometry of the ferroelectret's internal voids. Wang et al. [16] used a Su-8 photoresist mould on a silicon wafer to fabricate a polydimethylsiloxane (PDMS) ferroelectret with $50 \times 50 \times 50 \mu \mathrm{m}^{3}$ size cubic internal voids. This approach provided control over the dimensions of the internal voids in the ferroelectret but did not consider the effect of the geometry of the individual voids on the resulting piezoelectric properties. The demonstrated piezoelectric coefficient $d_{33}$ was $182 \mathrm{pC} / \mathrm{N}$, for the pure PDMS structure, which was about $40 \%$ smaller than the PP foam ferroelectret. A similar mould-casting approach fabricated PDMS ferroelectret with circular voids with a diameter of 100 $\mu \mathrm{m}$ and height of $40 \mu \mathrm{m}$ [17]. The $d_{33}$ of the fabricated ferroelectret was $350 \mathrm{pC} / \mathrm{N}$. However, the cross sectional geometry of the fabricated ferroelecteret is still rectangular shape, the other types of cross sectional geometry of the voids was also not modelled in this work.

In our previous work, we demonstrated the optimisation of a PDMS structure based on rectangular void for human body energy harvesting [18]. In this work, the numerical model has been applied to explore the effect of the microscale dimensions and a variety of void geometry on the properties of the ferroelectret. Based on this model, PDMS ferroelectrets with various void structures have been fabricated using silicon casting moulds made by MEMS fabrication techniques. This paper presents a summary of the theoretical predictions and the comparison of the simulations against the experimental results. An improved void structure of PDMS ferroelectret has been proposed using the model.

\section{Numerical algorithm and simulation}

To analyse the piezoelectric properties of a ferroelectret, a simplified model for a charge implanted cellular PDMS structure with micrometre-sized voids is illustrated in figure 1 [18-19]. 


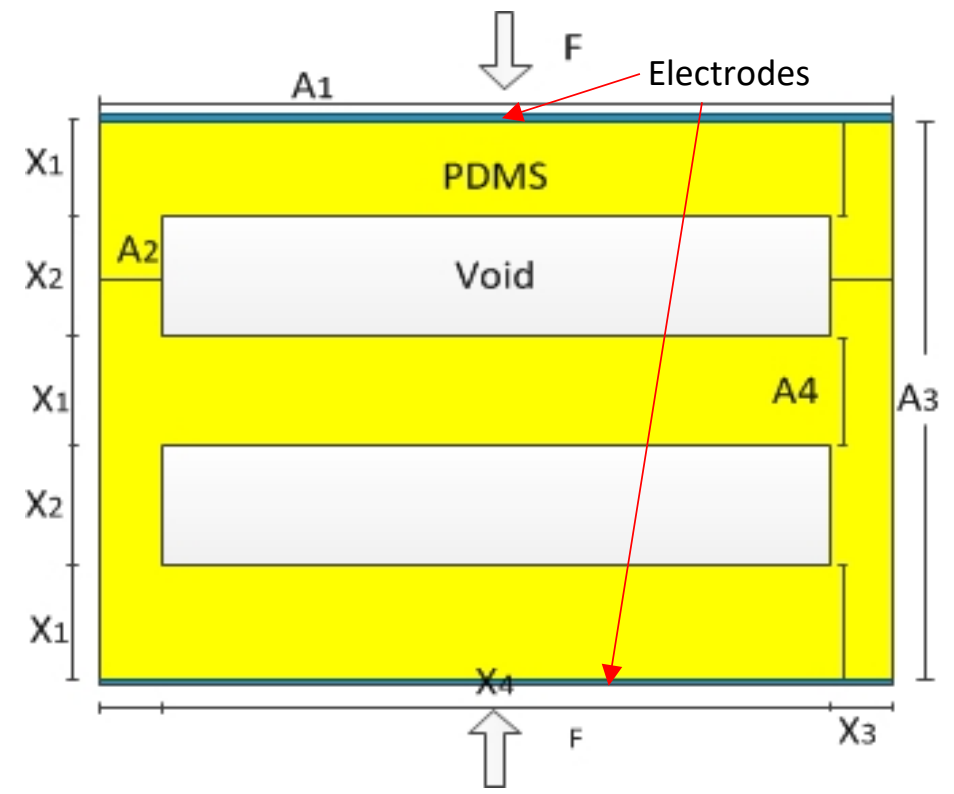

Figure 1. A simplified model for the piezoelectric properties of a charge-implanted cellular structure ( $x_{1}$ and $x_{2}$ are the thickness of the solid and void layers, respectively; $A_{1}$ and $A_{2}$ are the areas of the solid and void layers in horizontal direction, respectively; $A_{3}$ and $A_{4}$ are the areas of the solid and void layers in vertical direction, respectively; F represents the external force applied to the outer surface of the material) [19]

For a structure with $n$ void layers and $n+1$ solid layers, the electric field in the solid layers $\left(E_{1}\right)$ and void layers $\left(\mathrm{E}_{2}\right)$ can be obtained from Gauss' law for the interfaces:

$$
E_{1}=\frac{\sigma_{m}}{\varepsilon_{1} \varepsilon_{0}} \text { and } E_{2}=\frac{\sigma_{m}-\sigma}{\varepsilon_{2} \varepsilon_{0}}
$$

where $\sigma_{\mathrm{m}}$ is the charge density on the electrodes, $\sigma$ is the charge density on the void surface, and $\varepsilon_{0}, \varepsilon_{1}$ and $\varepsilon_{2}$ are the vacuum permittivity, the relative dielectric constant of PDMS and the relative dielectric constant of air $\left(\varepsilon_{2}=1\right)$, respectively.

From Kirchhoff's second law for short circuit conditions:

$$
V=\int E d x=(n+1) x_{1} E_{1}+n x_{2} E_{2}=0
$$

where $V$ is the electric potential across the electrode pair, and $x_{1}$ and $x_{2}$ are the thickness of the solid and void layers, respectively.

It is found that:

$$
\sigma_{m}=\frac{n \varepsilon_{1} \sigma x_{2}}{\left[(n+1) x_{1}+n \varepsilon_{1} x_{2}\right]}
$$

Therefore, the variation of the charge density on the electrodes $\left(\Delta \sigma_{\mathrm{m}}\right)$ can be expressed as a function of the thickness variation $\left(\Delta \mathrm{x}_{1}\right.$ and $\left.\Delta \mathrm{x}_{2}\right)$ by:

$$
\Delta \sigma_{m}=\frac{\partial \sigma_{m}}{\partial x_{1}} \Delta x_{1}+\frac{\partial \sigma_{m}}{\partial x_{2}} \Delta x_{2}=\frac{-n(n+1) \varepsilon_{1} \sigma x_{2}}{\left[(n+1) x_{1}+n \varepsilon_{1} x_{2}\right]^{2}} \Delta x_{1}+\frac{n(n+1) \varepsilon_{1} \sigma x_{1}}{\left[(n+1) x_{1}+n \varepsilon_{1} x_{2}\right]^{2}} \Delta x_{2}
$$

When an external force (F) deforms the multilayer structure, the relationships between the resulting stresses and strains can be expressed as: 


$$
\frac{F}{A_{3}}=c_{33} \frac{\Delta x_{3}}{x_{3}} \text { and } \frac{F}{A_{4}}=\frac{F}{s r_{2} A_{3}}=c_{33} \frac{\Delta x_{4}}{x_{4}}
$$

where $\mathrm{C}_{33}$ is the elastic modulus of PDMS, $\mathrm{A}_{3}$ and $\mathrm{A}_{4}$ are the areas of the solid and void layers, respectively and $s r_{2}$ is the ratio of $\mathrm{A}_{4}$ to $\mathrm{A}_{3}$.

The relationship between the thickness variations, $\Delta \mathrm{x}_{3}$ and $\Delta \mathrm{x}_{4}$, is therefore:

$$
\frac{\Delta x_{4}}{\Delta x_{3}}=\frac{1}{s r_{2}} \frac{x_{4}}{x_{3}}=\frac{t r_{2}}{s r_{2}}
$$

where $\operatorname{tr}_{2}$ is the ratio of $\mathrm{x}_{4}$ to $\mathrm{x}_{3}$,

Due to the Poisson effect, the relationships between the thickness variations, $\Delta \mathrm{x}_{3}$ and $\Delta \mathrm{x}_{2}, \Delta \mathrm{x}_{4}$ and $\Delta \mathrm{x}_{1}$, are therefore:

$$
\gamma \frac{\Delta x_{3}}{x_{3}}=\frac{\Delta x_{2}}{x_{2}} \text { and } \gamma \frac{\Delta x_{4}}{x_{4}}=\frac{\Delta x_{1}}{x_{1}}
$$

where $\gamma$ is the Poisson's ratio,

By substituting equation (6) into equation (7), the relationship between $\Delta \mathrm{x}_{2}, \Delta \mathrm{x}_{1}, \mathrm{x}_{2}$ and $\mathrm{x}_{1}$ is therefore:

$$
\Delta x_{1} x_{2}=\frac{1}{s r_{2}} \Delta x_{2} x_{1}
$$

By substituting equation (8) into equation (4), the variation of the charge density on the electrodes $\left(\Delta \sigma_{\mathrm{m}}\right)$ can be expressed as:

$$
\Delta \sigma_{m}=\frac{n(n+1) \varepsilon_{1} \sigma\left(s r_{2}-1\right) x_{2}}{\left[(n+1) x_{1}+n \varepsilon_{1} x_{2}\right]^{2}} \Delta x_{1}
$$

Since only a certain portion of the electrode surface is charged, the effective charge density $\left(\sigma_{\mathrm{m}}\right)_{\mathrm{eff}}$, and its variation, $\left(\Delta \sigma_{\mathrm{m}}\right)$ eff, should be:

$$
\left(\sigma_{m}\right)_{e f f}=\left(1-s r_{1}\right) \sigma_{m} \text { and }\left(\Delta \sigma_{m}\right)_{e f f}=\left(1-s r_{1}\right) \Delta \sigma_{m}
$$

where $A_{1}$ and $A_{2}$ are the effective areas of the solid and void layers, respectively, and $s_{1}$ is the ratio of $A_{2}$ to $A_{1}$.

By its definition, the piezoelectric coefficient $\left(\mathrm{d}_{31}\right)$ can be expressed as:

$$
d_{31}=\frac{\left(\Delta \sigma_{m}\right)_{e f f}}{F / A_{3}}=\frac{n(n+1) \varepsilon_{1} \sigma t r_{1}\left(1-s r_{1}\right)\left(s r_{2}-1\right) \gamma}{\left[(n+1)+n \varepsilon_{1} t r_{1}\right]^{2} c_{33}}
$$

where $t_{1}$ is the ratio of $\mathrm{x}_{2}$ to $\mathrm{x}_{1}$,

Because the piezoelectric coefficient $\left(\mathrm{d}_{33}\right)$ can be expressed as:

$$
d_{33}=\frac{\left(\Delta \sigma_{m}\right)_{e f f}}{F / A_{1}}=\frac{n(n+1) \varepsilon_{1} \sigma t r_{1}\left(1-s r_{1}\right)^{2}}{s r_{1}\left[(n+1)+n \varepsilon_{1} t r_{1}\right]^{2} c_{33}}
$$

Hence,

$$
\frac{d_{33}}{d_{31}}=\frac{1-s r_{1}}{s r_{1}\left(s r_{2}-1\right) \gamma}\left(0<s r_{1}, s r_{2}<1\right)
$$

Because $n, \varepsilon_{1}$ and $t r_{1}$ are always positive:

$$
(n+1)+n \varepsilon_{1} t r_{1} \geq 2 \sqrt{n(n+1) \varepsilon_{1} t r_{1} \varepsilon_{1}}
$$


The piezoelectric coefficient $\left(\mathrm{d}_{33}\right)$ can be simplified as:

$$
d_{33} \leq \frac{\left(1-s r_{1}\right)^{2} \sigma}{4 s r_{1} c_{33}}\left(0<s r_{1}<1\right)
$$

According to equation 12, the piezoelectric properties of a charged implanted, cellular polymer structure is determined by its geometry, elastic modulus and charge density. Since the elastic modulus and charge density of PDMS are constants in this work, the geometry of void can be investigated and its influence on the piezoelectric properties of the ferroelectret explored.

The mathematical analysis in equations (1) to (15) is only suitable for the basic rectangular voids shown in figure 1. More complex void geometries can be approximated to equivalent rectangles or must be simulated using FEA software to determine the void displacements. In order to investigate the effect of void geometry on void displacement, a rectangular, parallelogram, sector and isosceles trapezium geometries have been modelled (Figure 2). These were chosen because they can be arranged to have identical void areas $\left(\mathrm{A}_{2}\right)$, area ratio $\mathrm{sr}_{1}$ and thickness ratio $\mathrm{tr}_{1}$. The parallelogram, sector and isosceles trapezium void shapes were modelled as equivalent rectangles (shown in figure 2) and were simulated in ANSYS 2D. The overall three-dimensional structures for the four geometries are shown in Figure 3.

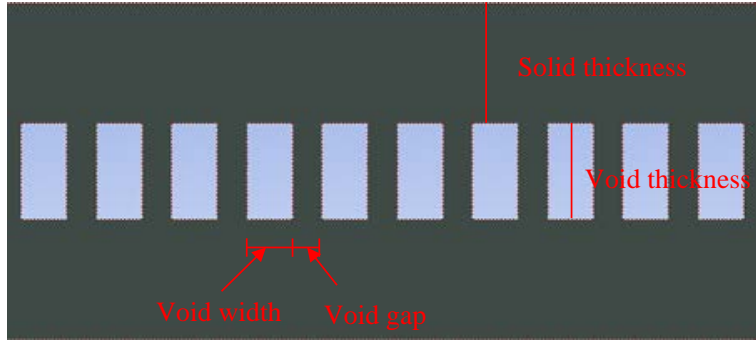

(a)

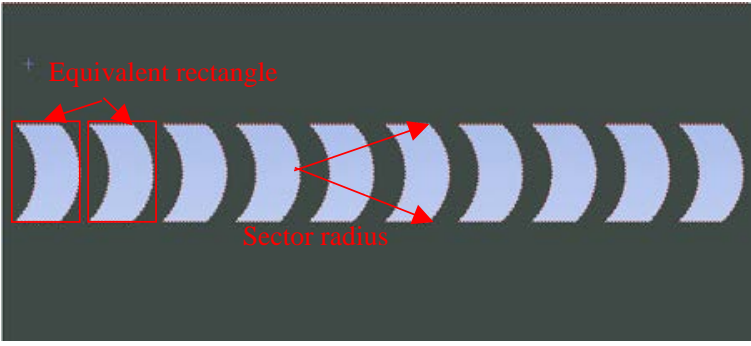

(c)

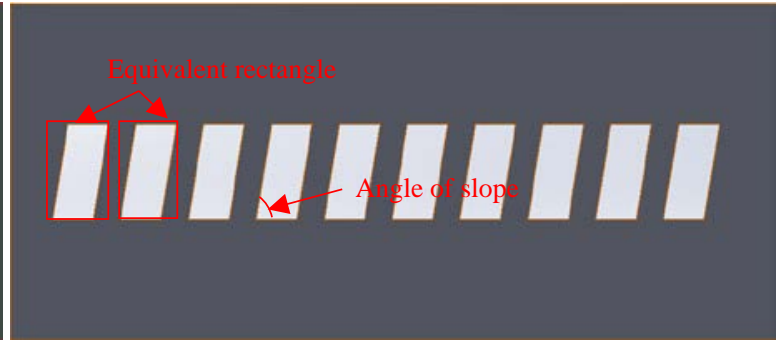

(b)

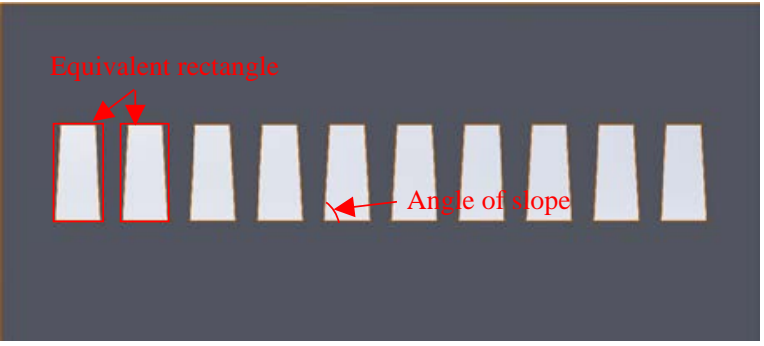

(d)

Figure 2. The structure of PDMS ferroelectret models with (a) rectangular voids, (b) parallelogram voids (c) sector shaped voids and (d) isosceles trapezium voids 

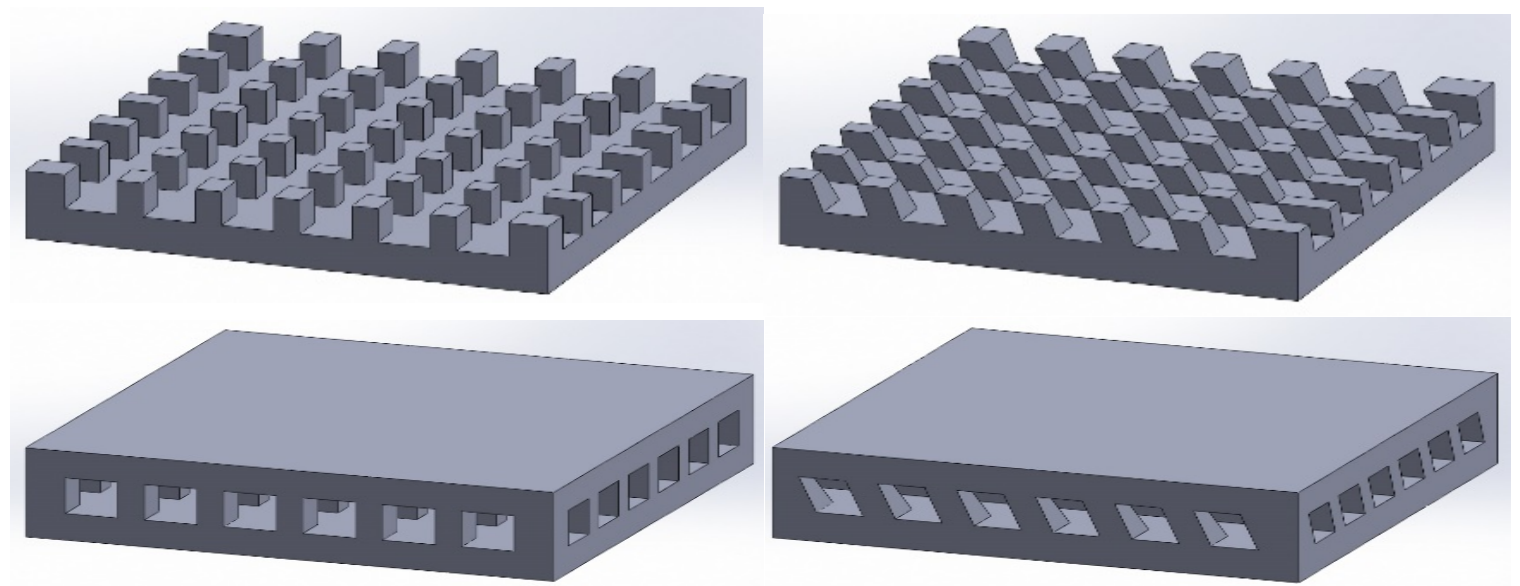

(a)

(b)
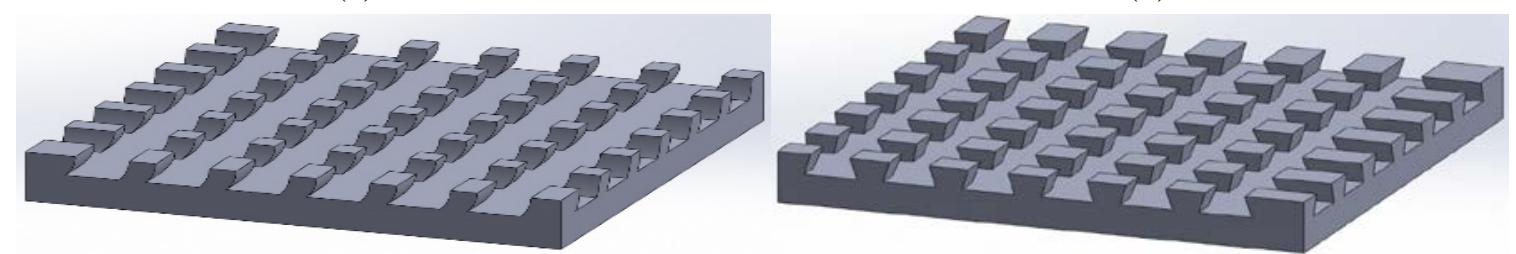

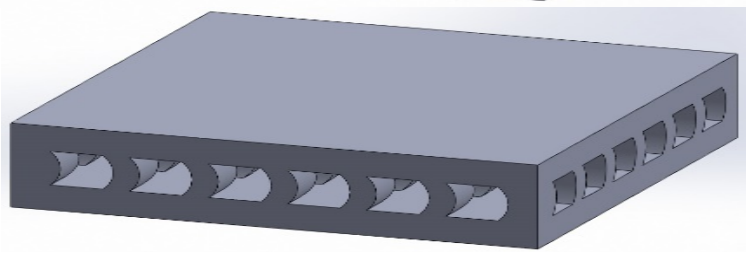

(c)

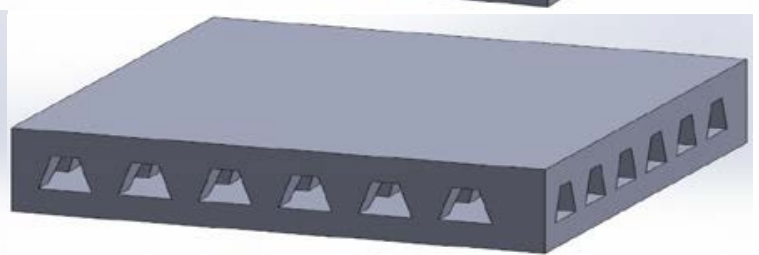

(d)

Figure 3. The cross sections and assembled ferroelectrets for (a) rectangular (b) parallelogram (c) sector (d) isosceles trapezium voids

All structures of PDMS are simulated in ANSYS 2D model, Figure 4(a) shows the 2D FEA mesh for the rectangular structure. Each structure was subjected to an external compressive mechanical load through the thickness of the material and ANSYS simulation used to determine the theoretical deformation of the voids. Assuming the charge density on the void surface is uniform, the variation in the net charge developed on the external surface can be calculated from the average deformation. In this case, the piezoelectric coefficient $d_{33}$ can be estimated by:

$$
d_{33}=\frac{\left(\Delta \sigma_{m}\right)_{e f f}}{F / A_{1}}
$$

By substituting equation (4) into equation (16), the piezoelectric coefficient $d_{33}$ can be expressed as

$$
d_{33}=2 \varepsilon_{1} \sigma_{m}\left(x_{2} \Delta x_{1}+x_{1} \Delta x_{2}\right)\left(2 x_{1}+\varepsilon_{1} x_{2}\right)^{-2} P^{-1}
$$

where $\Delta \mathrm{x}_{1}$ and $\Delta \mathrm{x}_{2}$ are the average variations of the thickness due to the applied external pressure $P$ calculated as shown in Figure $4(\mathrm{~b})$. The theoretical $d_{33}$ values for the 4 geometries calculated 
from the FEA (Equation 17) were compared with the results from the equivalent rectangle model, as shown in Figure 5.

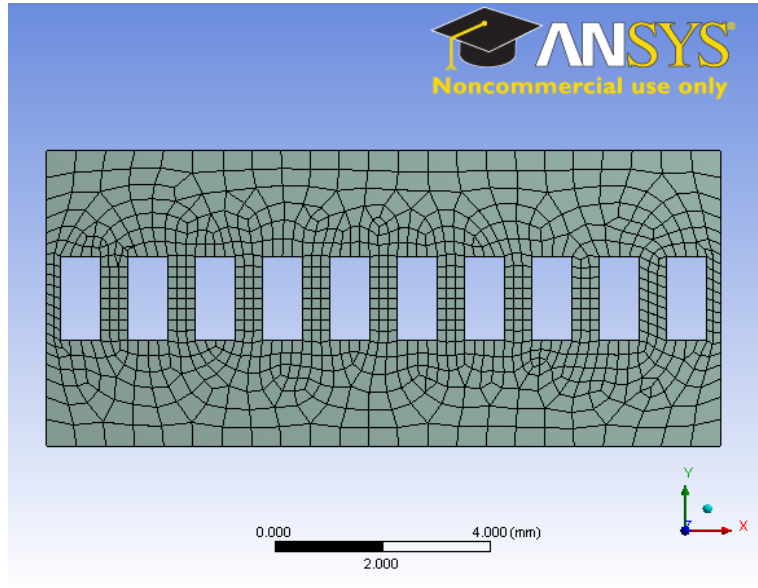

(a)

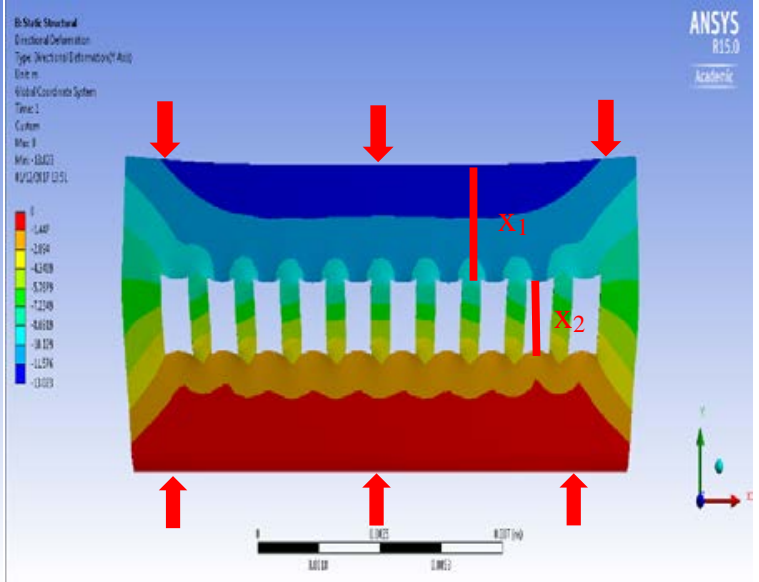

(b)

Figure 4. (a) 2D FEA mesh for the rectangular structure; (b) ANSYS simulation deformation results in thickness direction for single layer rectangle void ferroelectret

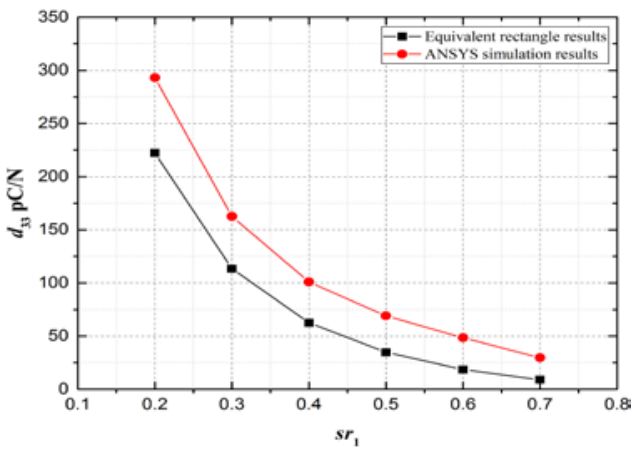

(a)

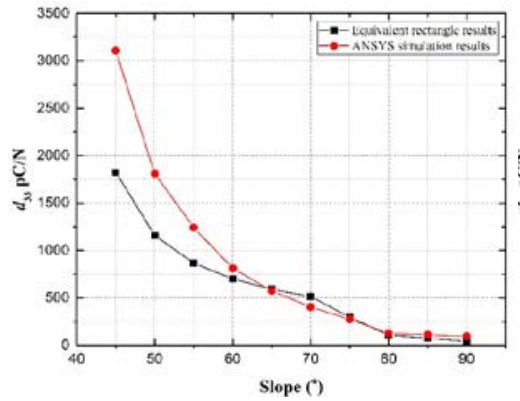

(c)

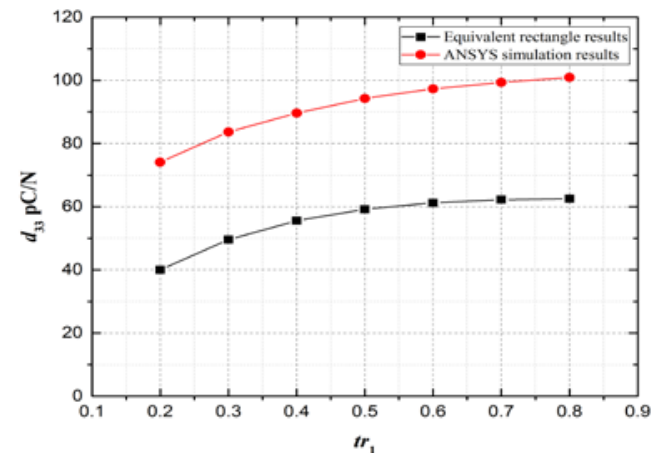

(b)

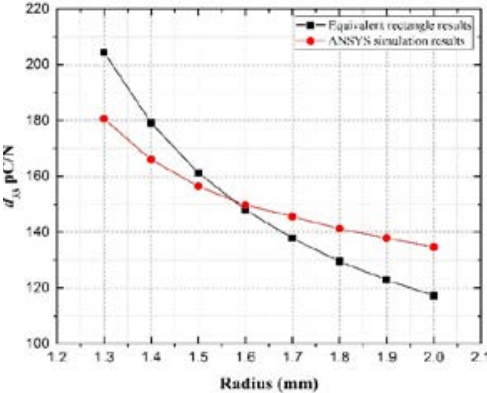

(d)

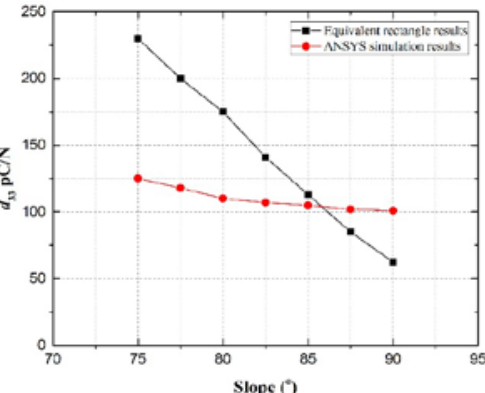

(e)

Figure 5. Equivalent rectangle results and ANSYS simulation results for $d_{33}$ from an applied pressure $1 \mathrm{kPa}$ : (a) rectangle voids versus with $s r_{1}$ with $t r_{1}=0.8$; (b) rectangle voids versus $t r_{1}$ with $s r_{1}=0.4$; (c) parallelogram voids varying with the angle of slope from $45^{\circ}$ to $90^{\circ}$ with $s r_{1}=0.4$ and 
$t r_{1}=0.8$; (d) sector voids varying with sector radius with $s r_{1}=0.4$ and $t r_{1}=0.8$; (e) isosceles trapezium varying with the angle of slope from $75^{\circ}$ to $90^{\circ}$ with $s r_{1}=0.4$ and $t r_{1}=0.8$

When the void is rectangular, Figure 5(a) and (b) shows that the $d_{33}$ increases significantly with the decreasing of $s r_{1}$ when $t r_{1}=0.8$, and increases slightly with the increasing of $t r_{1}$ when $s r_{1}=0.4$. In addition, from these results, there is a difference of $20 \%$ to $30 \%$ between the results from equivalent rectangle model and the ANSYS simulation. The difference is possibly caused by the fact that the analytical model only considers rigid deformation. The analytical model does not take into account any bending deformation of the material, resulting in the shape of void being unchanged.

For the parallelogram void geometry, $d_{33}$ varies with the angle of slope, which is the acute angle between the hypotenuse and the base, as shown in Figure 2(b). From Figure 5(c), the $d_{33}$ is a maximum at $45^{\circ}$ and this reduces with increasing angle until the parallelogram becomes a rectangle. For the sector void geometry, the $d_{33}$ versus radius is shown in Figure 5(d), which indicates $d_{33}$ decreases with the increasing sector radius. For the isosceles trapezium void geometry, $d_{33}$ varies with the angle of slope which is defined in Figure 2(d). From Figure 5 (e), the $d_{33}$ slightly increase with angle reducing from $90^{\circ}$ to $75^{\circ}$. For parallelogram and isosceles trapezium void geometry, the equivalent rectangle model results show an increasing disagreement with the ANSYS simulation results as the angle of the slope decreases. Of these four void geometries, the parallelogram void ferroelectret can achieve higher piezoelectric $d_{33}$ coefficient.

\section{Fabrication}

Due to Paschen's law, scaling down the thickness of the ferroelectret is a possible approach to enhancing the internal void surface charge density resulting in improved the piezoelectric coefficients $d_{33}$. The moulds used to fabricate the microscale rectangular and parallelogram voids were fabricated from silicon wafers using a bulk micromachining process, as shown in Figure 6. The rectangular mould was fabricated from a $<100>$ wafer whilst the parallelogram mould used a $<111>$ wafer. A layer of $2 \mu \mathrm{m}$ thick silicon dioxide was deposited on a blank silicon wafer by plasma-enhanced chemical vapour deposition (PECVD). Next, a $5 \mu \mathrm{m}$ thick of photoresist (AZ9260) was spin-coated on the silicon dioxide layer and then baked on a hotplate at $110{ }^{\circ} \mathrm{C}$ for 120 seconds. The mould pattern was defined using standard contact lithography with a 10 second exposure followed by development in AZ400K for 6 minutes. An inductively coupled plasma (ICP) etch was used to etch the exposed silicon dioxide and this was followed by stripping the resist leaving the patterned silicon dioxide masking layer. The wafer then dip etched in hydrofluoric acid (HF) to remove the natural silicon dioxide and the silicon was deep reaction ion etched (DRIE) to a depth of $50 \mu \mathrm{m}$. On the $<100$ wafer this formed the rectangular mould and the process is completed by stripping the remaining silicon dioxide. For the $<111>$ wafer, the parallelogram

structure was formed by following the DRIE etch with a wet anisotropic potassium hydroxide $(\mathrm{KOH})$ etch. The anisotropic nature of the etch converts the rectangular structure into a parallelogram cross-section with an angle of slope of $70.5^{\circ}$ as defined by the crystalline planes [20]. The top view of the fabricated rectangular and parallelogram moulds are shown in Figure 7(a) and 7(b), respectively. The cross-section view of the fabricated rectangular and parallelogram void silicon mould are shown in Figure 7(c). 


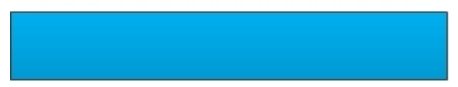

(a) 4 inch Si wafer

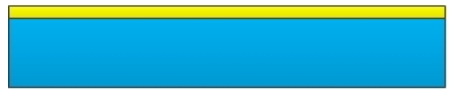

(b) $\mathrm{SiO}_{2}$ deposition

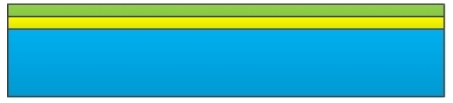

(c) Apply photoresist

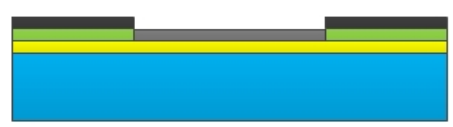

(d) Lithography

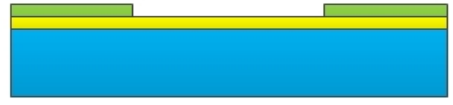

(e) Develop resist

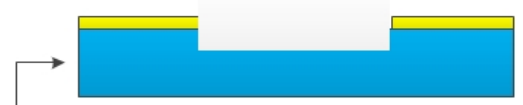

(1) Strip resist

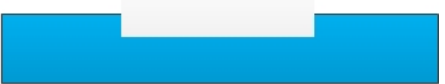

(2) HF etch

(f) $\mathrm{SiO}_{2}$ DRIE etch

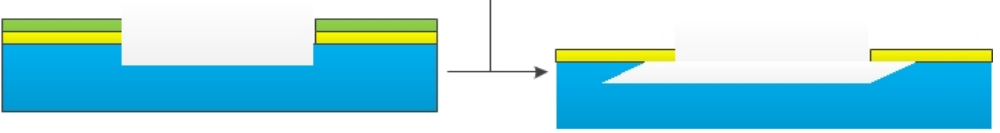

(g) Si DRIE etch

(1) $\mathrm{KOH}$ etch

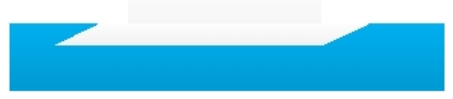

(2) HF etch

$<111>$ parallelogram void wafer

Figure 6. Schematic of silicon mould fabrication processes for $<100>$ rectangular void and $<111>$ parallelogram void wafer.

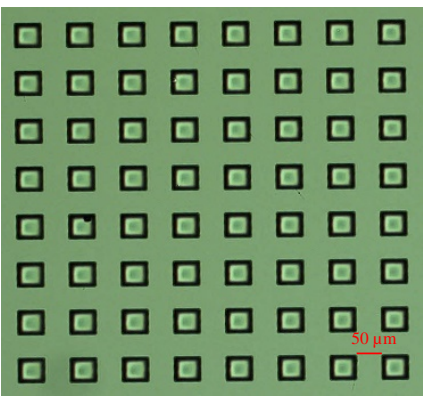

(a)

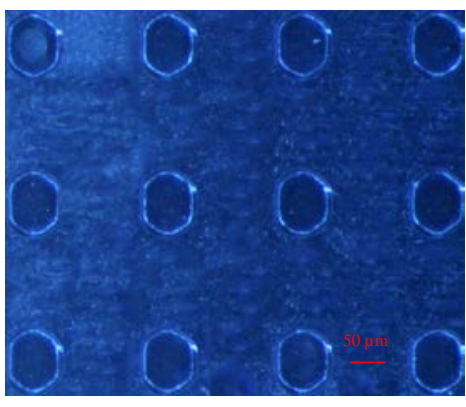

(b)

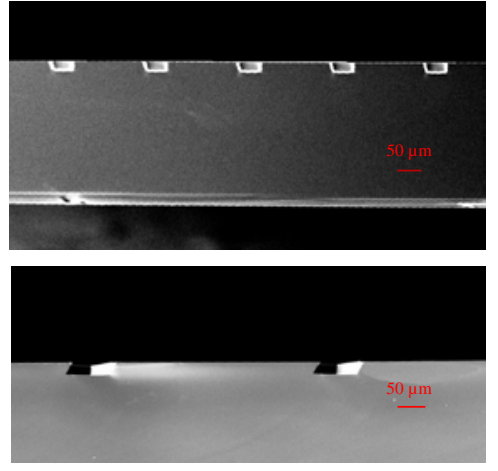

(c)

Figure 7. (a) The optical microscope image of top view of the fabricated rectangular mould; (b) the optical microscope image of top view of the fabricated parallelogram mould; (c) the SEM image of cross-section view of fabricated rectangular and parallelogram void silicon mould

To experimentally evaluate the piezoelectric properties of the ferroelectret at different area ratios, three rectangular and parallelogram void structures with area ratios of $0.25,0.16$ and 0.0625 were explored. Silicon moulds with three different depths $(30 / 40 / 50 \mu \mathrm{m})$ were fabricated to evaluate the effect of the thickness ratio. A summary of the main parameters of the fabricated samples is shown on the table below: 
Table 1. A summary of the parameters the fabricated ferroelectret

\begin{tabular}{|c|c|c|c|c|c|}
\hline Void shape & $t_{1}$ & $s r_{1}$ & $\begin{array}{c}\text { Void } \\
\text { Thickness }(\mu \mathrm{m})\end{array}$ & $\begin{array}{c}\text { Void width } \\
(\mu \mathrm{m})\end{array}$ & $\begin{array}{c}\text { Void gap } \\
(\mu \mathrm{m})\end{array}$ \\
\hline Rectangular & $0.4-0.8$ & $0.25 / 0.16 / 0.0625$ & $30 / 40 / 50$ & $50 / 75 / 150$ & 50 \\
\hline Parallelogram & $0.4-0.8$ & $0.25 / 0.16 / 0.0625$ & $30 / 40 / 50$ & $46.3 / 71.3 / 146.3$ & 53.7 \\
\hline
\end{tabular}

The etched silicon wafer can not be used immediately as a mould because the PDMS adheres too strongly to the surface making removal problematic. To prevent this, the fabricated silicon mould was placed in a desiccator under vacuum for $1 \mathrm{~h}$ with a glass sheet containing a few drops of tricholro $(1 \mathrm{H}, 1 \mathrm{H}, 2 \mathrm{H}, 2 \mathrm{H}$-perfluorooctyl)silane (Sigma Aldrich, MO, USA) to silanize its surface [21].

The PDMS layer was fabricated by mixing a liquid PDMS and curing agent (Sylgard 184 from Dow Corning, MI, USA) at a ratio of 10:1 by weight and then degassed in a vacuum desiccator. To remove the moisture on the mould, the wafer was cleaned by acetone and oven baked for 15 minutes at $120^{\circ} \mathrm{C}$. A thin PDMS mixture layer was spin-coated on the silicon wafer mould and cured for 1 $\mathrm{h}$ at $80^{\circ} \mathrm{C}$. The thickness of the PDMS membrane was controlled by the speed and duration of the spin-coating [22]. For this experiment, the duration of spin-coating was fixed at 30 seconds. To achieve the designed thickness ratio, a summary of the spin-coating speeds is listed in table 2 . The mould was immersed in water and the finished thin PDMS membrane was peeled away from the mould. This process was repeated using a plain unetched silicon wafer to produce an unstructured smooth PDMS layer. Both PDMS films were then given an oxygen plasma treatment (Femto Asher, Diener, Germany, $30 \mathrm{~S}$ at 35-40 W). Immediately after the plasma treatment, the two PDMS layers were bonded together by applying a gentle pressure. The bonded PDMS assembly was baked in oven at $80^{\circ} \mathrm{C}$ for 1 hour to improve the bond strength [23]. Multi-layered samples were constructed by repeating the processes.

Table 2. A summary of the speed of the spin coating

\begin{tabular}{|c|c|c|c|}
\hline $\operatorname{tr}_{1}$ Void thickness & $30 \mu \mathrm{m}$ & $40 \mu \mathrm{m}$ & $50 \mu \mathrm{m}$ \\
\hline 0.4 & $1145 \mathrm{rpm}$ & $890 \mathrm{rpm}$ & $730 \mathrm{rpm}$ \\
\hline 0.5 & $1390 \mathrm{rpm}$ & $1080 \mathrm{rpm}$ & $890 \mathrm{rpm}$ \\
\hline 0.6 & $1630 \mathrm{rpm}$ & $1270 \mathrm{rpm}$ & $1045 \mathrm{rpm}$ \\
\hline 0.7 & $1870 \mathrm{rpm}$ & $1450 \mathrm{rpm}$ & $1195 \mathrm{rpm}$ \\
\hline 0.8 & $2100 \mathrm{rpm}$ & $1630 \mathrm{rpm}$ & $1340 \mathrm{rpm}$ \\
\hline
\end{tabular}


The fabricated PDMS samples $(2 \mathrm{~cm} \times 2 \mathrm{~cm}$ ) were corona charged, which generated macroscopic dipoles by the gas-breakdown inside the voids during charging. The corona charging needle was located with its tip about 3-4 cm above the sample surface. The PDMS was charged with a needle voltage of $-25 \mathrm{kV}$ for 2 minutes at room temperature. The external electrodes were formed using adhesive silver tape.

Figure 8 and 9 show the cross-section image of the fabricated ferroelectret membranes with rectangular and parallelogram voids, and the multilayer structures, respectively. From these figures, it can observed that the designed void shape was successfully fabricated and have excellent uniformity. However, it found that the voids are not aligned vertically in the multilayer ferroelectrets.

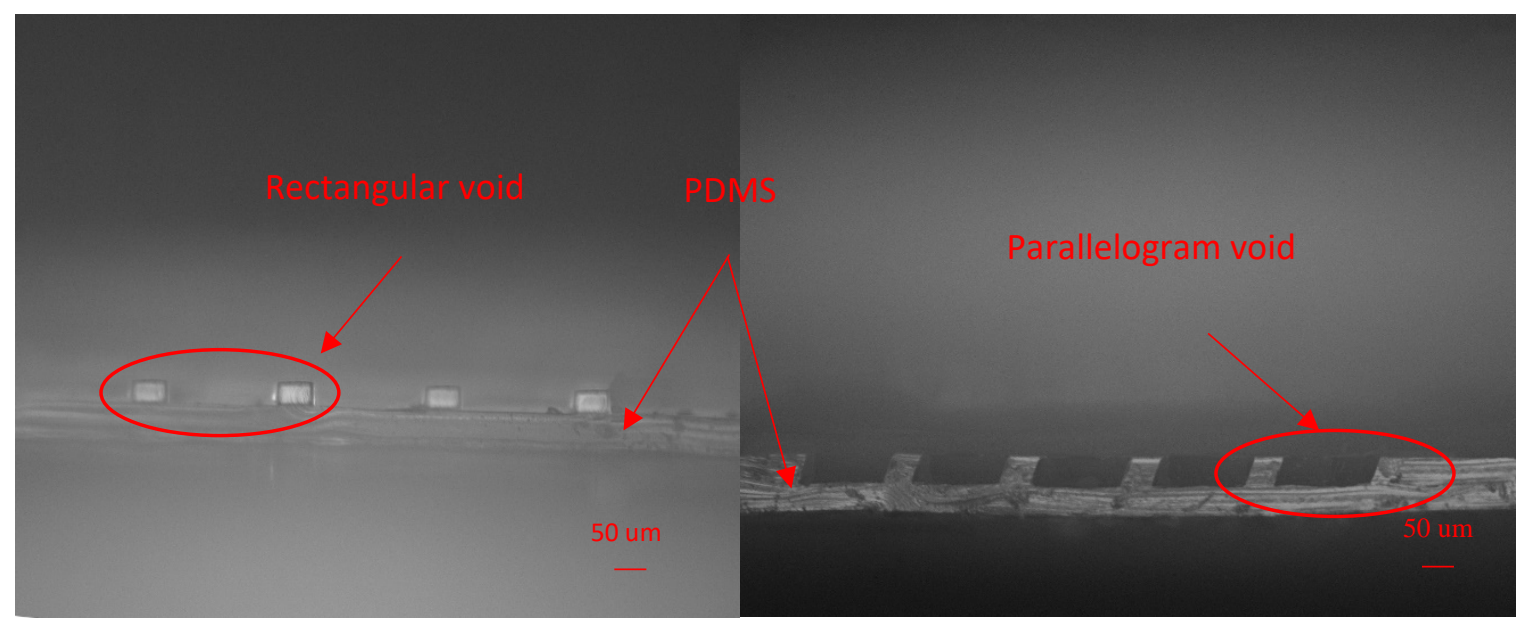

(a)

(b)

Figure 8. The SEM image of the cross section image for fabricated (a) rectangular void and (b) parallelogram void PDMS ferroelectret membrane 


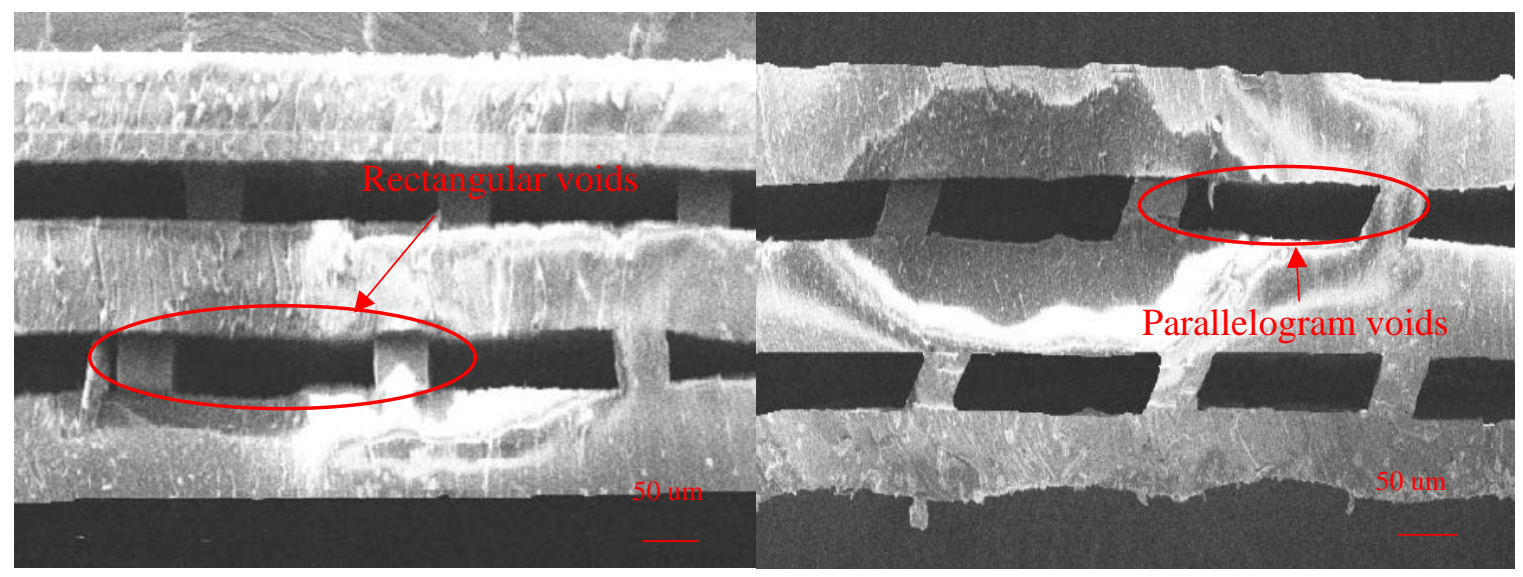

(a)

(b)

Figure 9. The image of the cross section for multilayer PDMS ferroelectret (a) rectangular PDMS ferroelectret (b) parallelogram void PDMS ferroelectret

\section{Experimental validation}

\subsection{Experimental step}

To experimentally validate the simulated results, rectangular and parallelograms voids with the same effective area ratio were fabricated using the silicon moulds. The top view of the rectangular and parallelogram void structures were square-shaped and hexagon-shaped, respectively (Figure 7). In order to achieve the same effective area ratio, the area of hexagon should be equal to the area of the square.

To investigate the energy harvesting performance of the PDMS ferroelectret, the experiment was carried on immediately after polarizing the samples. An Instron electrodynamic instrument (EletroPuls E1000, Instron Ltd) was used to apply a repeatable force profile that simulates the forces applied during walking. The average step frequency of a human walking is in the range of 0.4 to 1.45 step per second [24-26]. Three compressive force profiles were applied with a maximum force of $800 \mathrm{~N}$ and their waveforms are shown in Figure 10. The output voltages of the PDMS ferroelectret under these compressive forces were recorded using an oscilloscope (TDS2014, Tektronix UK Ltd) for different load resistances from $1 \mathrm{M} \Omega$ to $1 \mathrm{G} \Omega$. 


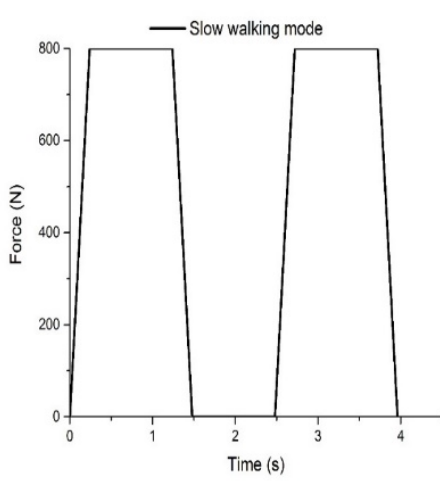

(a)

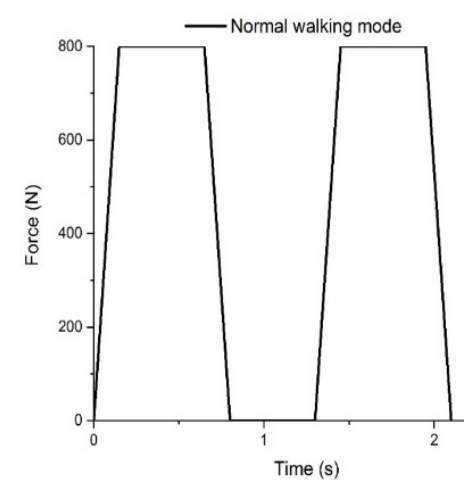

(b)

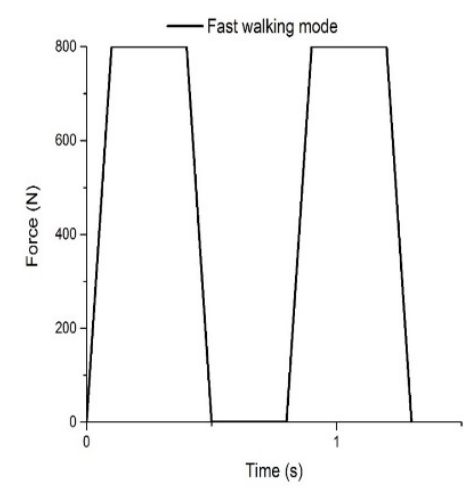

(c)

Figure 10. Waveform of applied compressive force at (a) slow walk at $0.4 \mathrm{step} / \mathrm{s}: 0.24 \mathrm{~s}$ transition time of the applied force from 0 to $800 \mathrm{~N}$ and duration of the maximum force was $1 \mathrm{~s}$; (b) medium walking speed at $0.8 \mathrm{step} / \mathrm{s}$, transition time $0.15 \mathrm{~s}$ and duration of the maximum force $0.5 \mathrm{~s}$; (c) fast walk at $1.4 \mathrm{step} / \mathrm{s}$, transition time $0.1 \mathrm{~s}$, duration of the maximum force $0.3 \mathrm{~s}$.

\subsection{Result and discussion}

\subsubsection{Piezoelectric coefficients measurement}




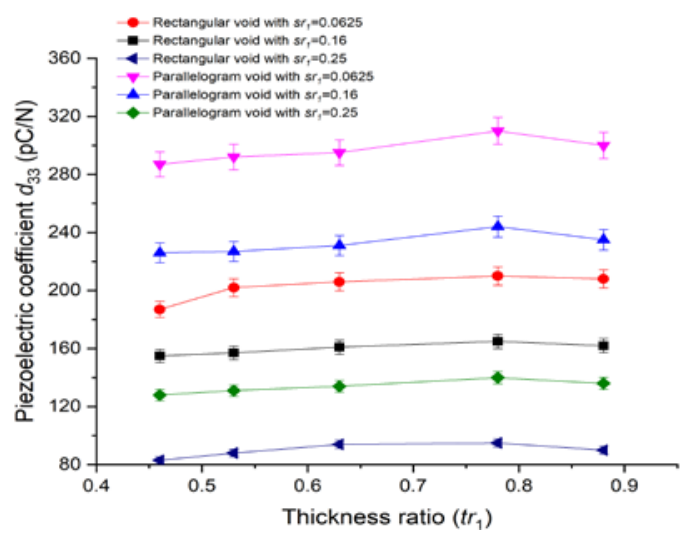

(a)

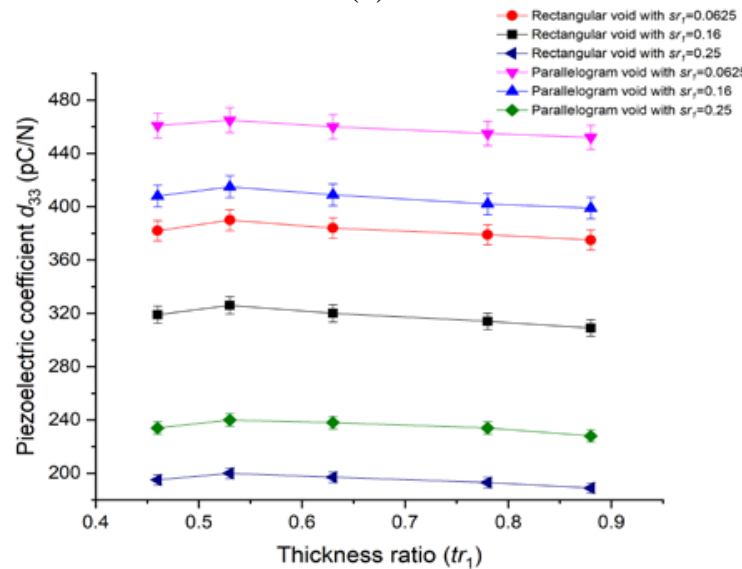

(c)

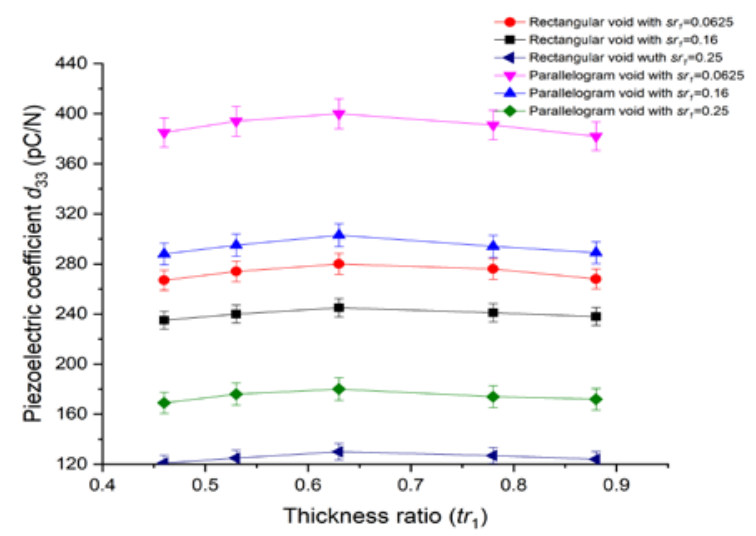

(b)

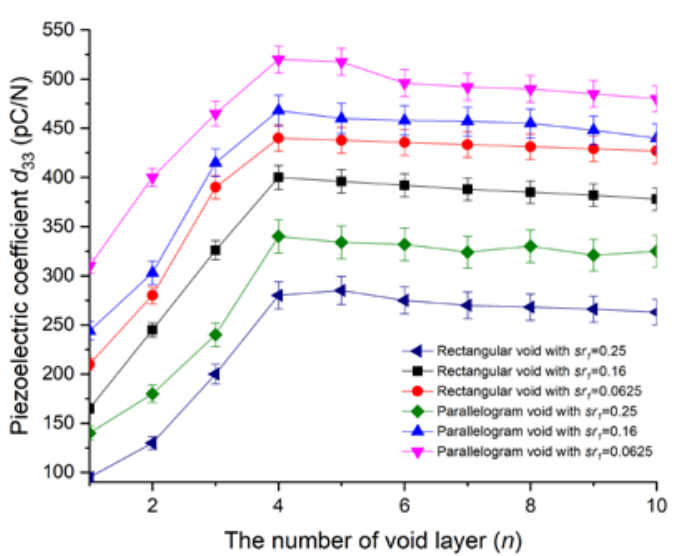

(d)

Figure 11. The measured piezoelectric coefficient varying with the thickness ratio for (a) one void layer, (b) two void layers and (c) three void layers; (d) The measured piezoelectric coefficient varying with the number of void layers with optimum $t r_{1}$ for each geometry.

Results of piezoelectric coefficient $d_{33}$ varying with the thickness ratio for PDMS ferroelectret with one, two and three void layers are shown in Figure 11(a)-(c), respectively. The piezoelectric charge constant $d_{33}$ is measured using a PiezoMeter PM300 (Piezotest Ltd). For this measurement, the measurement setting was a dynamic force of $0.25 \mathrm{~N}$, static force of $10 \mathrm{~N}$ and frequency of $110 \mathrm{~Hz}$. It is found that the piezoelectric confident $d_{33}$ of both samples increase with the thickness ratio. The best piezoelectric properties for one, two and three void layers PDMS ferroelectret are achieved around the thickness ratio of $0.8,0.6$ and 0.55 , respectively. It is also found that the effective area ratio does affect the resulting piezoelectricity. With the number of void layer increasing, the variations of piezoelectric properties were shown in Figure 11(d). When stacking more void layers together up to 4 layers, it is found that $d_{33}$ of PDMS ferroelectret have a significant improvement. The maximum piezoelectric coefficients $d_{33}$ are achieved by 4-layer PDMS ferroelectret, about 520 $\mathrm{pC} / \mathrm{N}$. When 5 or more layers are stacked, $d_{33}$ does not increase any further. This may be due to an increase in Young's modulus of the ferroelectrets as the number of void layers increases, resulting in a decrease in piezoelectricity [28]. Table 3 provides the piezoelectric coefficient values of different ferroelectret material in order to compare them with the fabricated PDMS reported in this work. The fabricated PDMS exhibits an important piezoelectric properties compared to other ones. 
Table 3. Piezoelectric coefficient values for piezoelectric polymer materials

\begin{tabular}{|l|c|c|}
\hline Piezoelectric material & $d_{33}(\mathrm{pC} / \mathrm{N})$ & $\begin{array}{c}\text { Young's modulus } \\
(\mathrm{kPa})\end{array}$ \\
\hline Cellular PDMS (Our current work) & 240 & 670 \\
\hline Cellular PDMS [17] & 350 & 300 \\
\hline Cellular PDMS [19] & 32.8 & 500 \\
\hline Cellular PP [29] & 200 & 1500 \\
\hline PTFE/FEP multilayer VCP [30] & 300 & 2400 \\
\hline
\end{tabular}

For ANSYS simulation results, the predicted piezoelectric coefficient $d_{33}$ of rectangular and parallelogram one void layer PDMS ferroelectret with $s r_{1}=0.16$ and $t r_{1}=0.8$ are around 100 and 320 $\mathrm{pC} / \mathrm{N}$, respectively. The experimental $d_{33}$ of the rectangular and parallelogram void single layer PDMS ferroelectret are 160 and $250 \mathrm{pC} / \mathrm{N}$, respectively (Figure 11(d)). The measured effective elastic modulus is about $670 \mathrm{kPa}$. Whilst the predicted values may be different, both the simulated and practical results show the parallelogram voids give higher piezoelectric properties than the rectangular voids. The difference between the simulation and experimental results is potentially due to the analytical calculation of $d_{33}$ which assumes identical charge distribution for the parallelogram and rectangular voids. Results of simulated electric field distribution for parallelogram void and rectangular void with identical charge distribution condition are shown in Figure 12 (a)-(b), respectively. It is found the electric field for the overlapping area of the parallelogram void with the upper and lower siders is uniformly distributed, as same as the electric field distribution of rectangular void. In contrast, the electric field distribution in the region between the ends of the parallelogram void is non-uniformly distributed. In other word, the actual charge distribution in parallelogram void should not be identical. However, the established model is based on the assumption that the internal electric field is evenly distributed. Therefore, the experimental results and theoretical results have certain errors. Also the calculation of area ratio for the parallelogram is identical to the rectangular structure and this may not be as accurate given the sloping sidewalls of the parallelogram structure. 


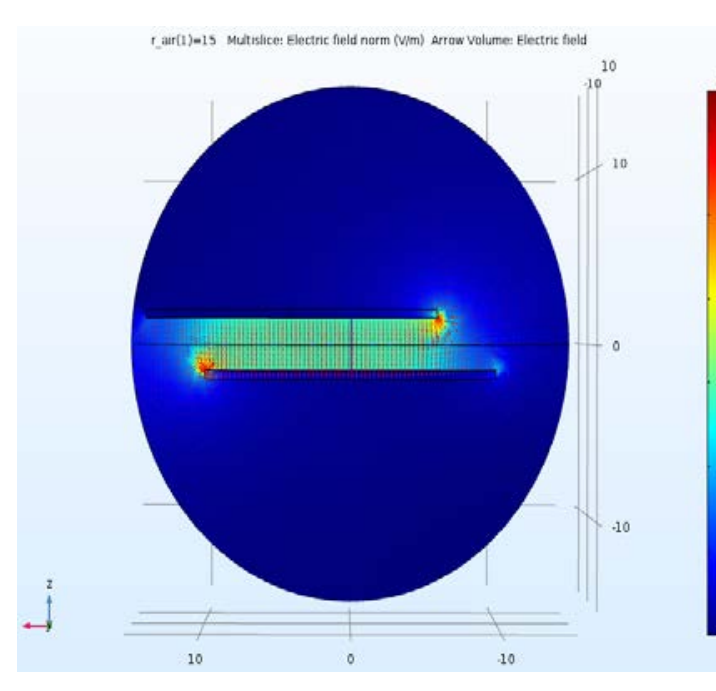

(a)

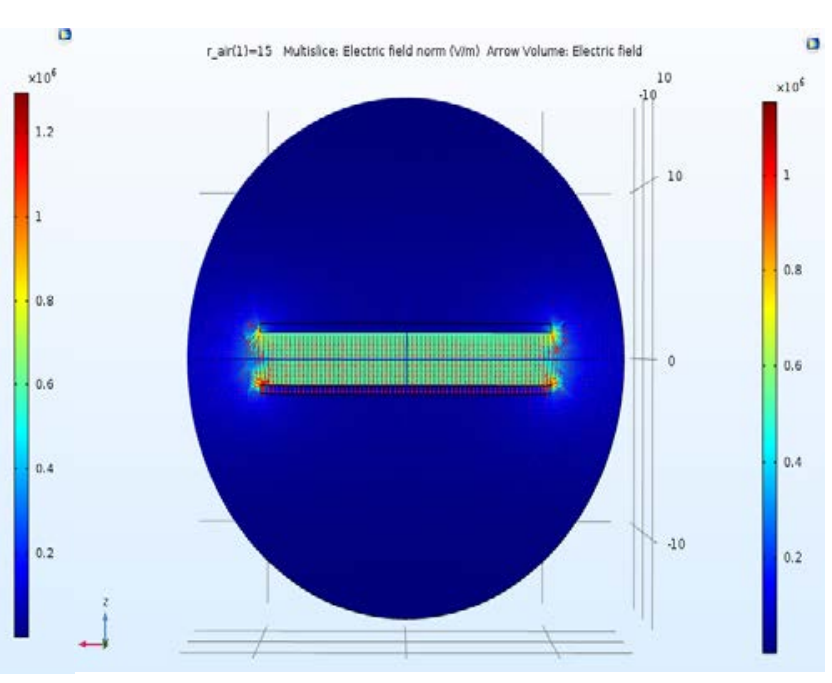

(b)

Figure 12. The simulated electric filed distribution for identical charge distribution on (a) parallelogram void and (b) rectangular void.

\subsubsection{Investigation of energy harvesting performance}

Figure 13 (a) shows the characteristic current-voltage ( $I-V)$ curves of a four layers parallelogram PDMS ferroelectret under compressive forces that were applied in normal walking mode, as a function of load resistance. The voltage values used in Figure 13 (a) are the average of measured voltages from three consecutive pulses. The peak current decreases with increasing load resistance in the range of $1 \mathrm{M} \Omega$ to $1 \mathrm{G} \Omega$. The voltage increases with increasing load resistance. Consequently, the power output reaches a maximum value of $2.73 \mu \mathrm{W}$ with a $65 \mathrm{M} \Omega$ loading resistance as shown in Figure 13 (b). The energy output of the fabricated PDSM ferroelectret is showing output peak power density of $0.68 \mu \mathrm{W} / \mathrm{cm}^{2}$ in normal walking mode. Compared to the commercial pp materials output peak density of $0.34 \mu \mathrm{W} / \mathrm{cm}^{2}$, there is a significant improvement [33]. Due to impedance matching theory, to maximize the output power, the output impedance should be equal to the input impedance of ferroelectret. The input impedance of the ferroelectret can be simplified as impedance of a capacitor. The input impedance of the ferroelectret can be expressed as:

$$
X_{c}=\frac{1}{2 \pi f C_{f}}=R_{L}
$$

Where $X_{\mathrm{c}}$ is the impedance of the ferroelectret, $f$ is the frequency of the output current, $C_{f}$ is the capacitance of the ferroelectret and the $R_{\mathrm{L}}$ is the output impedance. 


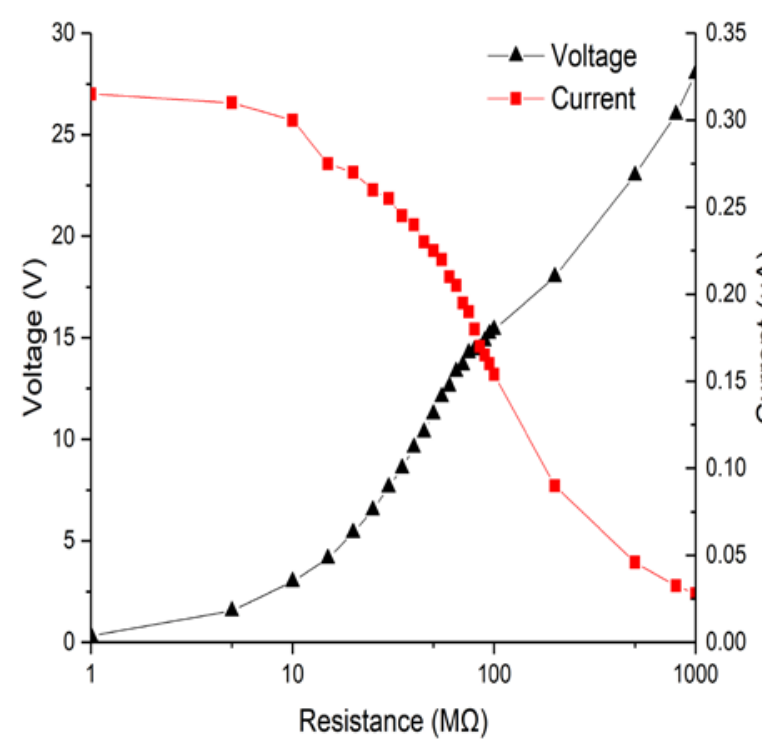

(a)

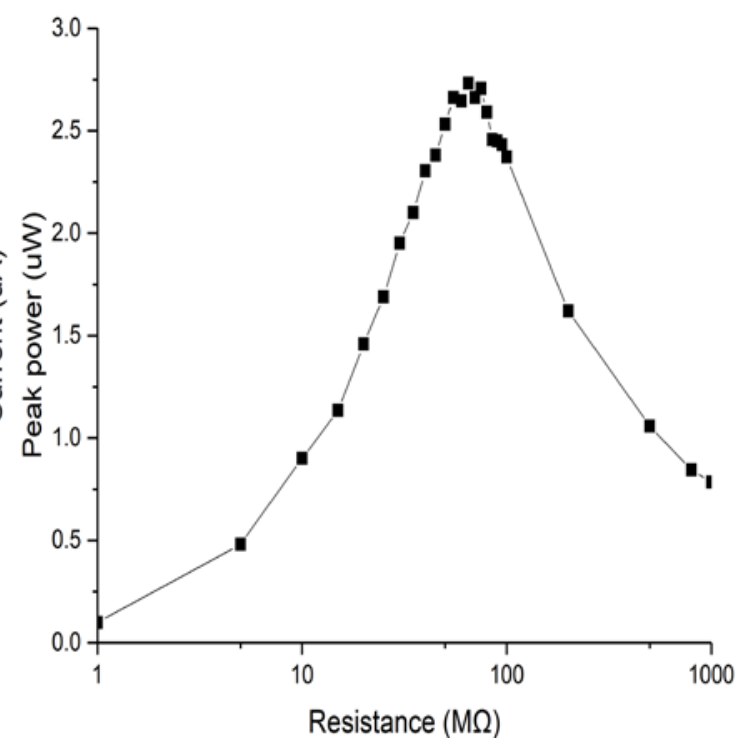

(b)

Figure 13. (a) The characteristic $I-V$ curves of a four layers PDMS ferroelectret; (b) Instantaneous power output at different resistance loading

In energy harvesting applications where the energy is extracted in pulses it may be preferable that the power management circuit is self-powered [27], i.e. the generated energy is used straightway and no energy storage is used. The advantage of powering from the input comparing to powering from the system energy storage is that the power management circuit is active only during the energy pulse, and thus the overall quiescent losses are minimized. However, the power losses of the power management circuit are strongly influenced by the supply voltage [28, 31]. Therefore, the high voltage generated by the proposed ferroelectret may not be desirable for the electronic circuit. It may also be desirable to increase the pulse width at the expense of energy content in order to reduce the intermittency, which in itself causes system energy loss during start-up, as shown in [32]. In addition, as many integrated circuits only work safely under a voltage stress of $5.5 \mathrm{~V}$, and a high external resistive load to the materials will make the matching from power converter being difficult in the CMOS process, it is ideal to have output voltage in the range of $1 \mathrm{~V}$ to $5.5 \mathrm{~V}$. At 10 $\mathrm{M} \Omega$, the output peak voltage of the ferroelectret is $3 \mathrm{~V}$ and its instantaneous current is $0.3 \mu \mathrm{A}$, which, once rectified and smoothed, would be suitable to be used directly as the supply to electronic circuits. Thus $10 \mathrm{M} \Omega$ is used as the resistive load in the following measurements.

The maximum compressive force applied on the ferroelectret in this work is set to be $800 \mathrm{~N}$, roughly resembling a walking person with weight of $80 \mathrm{~kg}$ in normal walking mode. The energy output is investigated in this work by varying the quantities of compressive forces. Figure 14 (a) shows the change of peak output voltage at different applied forces with load resistance of $10 \mathrm{M} \Omega$. It is found that the output voltage increases linearly with increasing forces. 


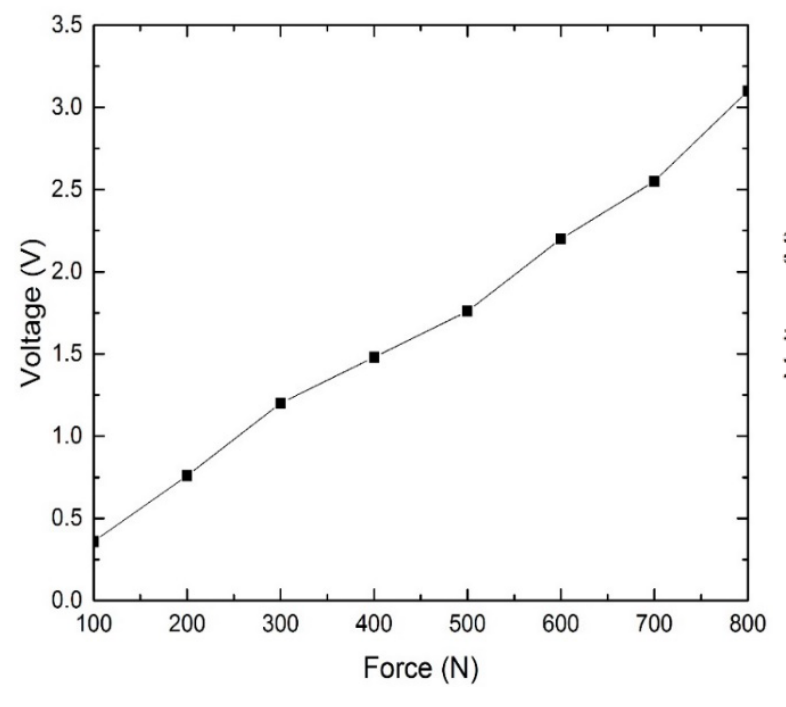

(a)

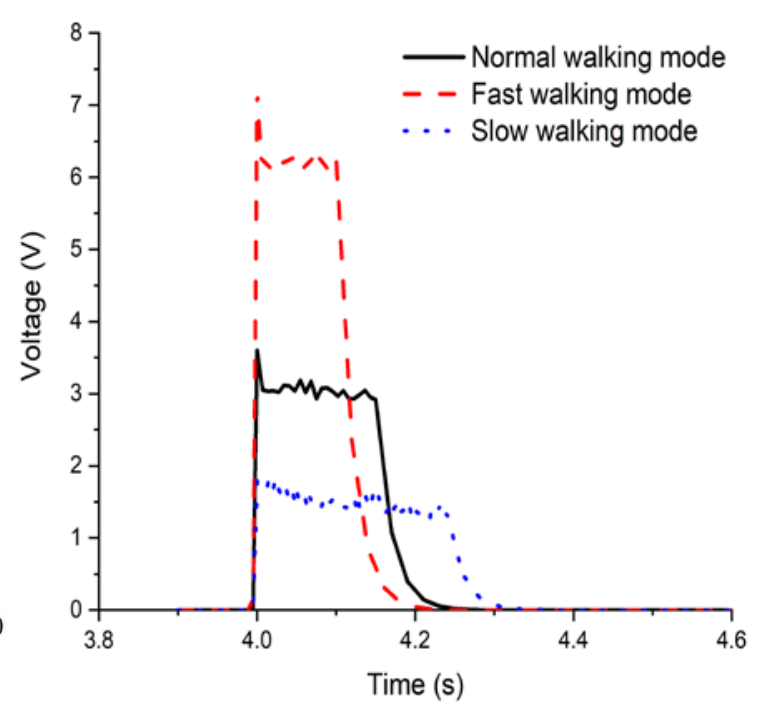

(b)

Figure 14. (a) Peak output voltage at different applied compressive forces (b) Output voltage pulse for three different model under a constant force of $800 \mathrm{~N}$

The output voltage is also affected by the walking mode. Figure 14 (b) shows the single output pulses of PDMS ferroelectret under $800 \mathrm{~N}$ compressive force in different walking modes. The step frequency of the slow walking mode, normal walking mode and fast walking mode are $0.4 \mathrm{step} / \mathrm{s}$, $0.8 \mathrm{step} / \mathrm{s}$ and $1.4 \mathrm{step} / \mathrm{s}$, respectively. From this Figure, the voltage peak increase with the increasing step frequency and the maximum voltage peak is achieved in the fast walking mode with the highest step frequency. However, the duration of voltage output pulses is decreased with the increasing step frequency. This can be explained by considering the change of force. In these walking modes with maximum force of $800 \mathrm{~N}$, the applied compressive force can be considered as an object with a velocity. During the whole collision process, the deformation of sample is regarded as an elasticity deformation. Due to Hooke's law, the applied force on the sample can be expressed as:

$$
F=-k X
$$

Where $F$ is the applied force, $k$ is a spring constant and $X$ is the deformation of sample.

Thus the force $F$ is directly proportional to $X$. With the step frequency increasing, the slope of applied force is increasing resulting in increasing deformation of the sample in unit time. As mentioned in previous chapter, the generated charge is directly proportional to the deformation of sample as:

$$
V=\frac{\Delta Q}{t} R
$$

Where $V$ is the output voltage, $\Delta Q$ is the variation of charge, $t$ is the time and $R$ is the load resistance.

For these three walking modes, the applied force are the same resulting in the final deformation of sample should be the same. Thus the amount of variation of charge should be the same. Form Eq. (20), the peak voltage $V$ of an output pulse is directly proportional to the duration of the applied force. It means that the peak voltage increase with the slope of applied force increasing. This explain the difference in voltage output in different walking modes. For the fast walking mode, it has the shortest transition time of the applied force from 0 to $800 \mathrm{~N}$ resulting in the maximum 
output peak voltage. Comparing with output pulses and the applied force wave function, the duration of output pulses is determined by transition time of the applied force. In addition, with the duration of force holding increasing, the duration of output pulses cannot be enhanced.

\section{Conclusions}

This paper presents a numerical FEA model to describe ferroelectret materials and to further improve their piezoelectric properties. Based on this FEA model, multilayer ferroelectret material with rectangular and parallelogram void structure were designed and fabricated utilizing silicon fabrication processes. There is a large error between the simulated and measured results, because the FEA software (ANSYS) lack ferroelectret model that can be used directly for predicting the energy performance and output. The FEA model only was used for guiding the design of the ferroelectret structures based on static structural model. However, the practical experiment and numerical results still revealed that the PDMS ferroelectret with lower effective area ratio $s r_{1}$ can generate higher piezoelectric coefficients $d_{33}$ and the parallelogram void PDMS ferroelectret based on the same effective area ratio can achieve higher piezoelectric coefficients $d_{33}$ than the rectangular void PDMS ferroelectret. The thickness ratio can also affect the piezoelectric coefficient $d_{33}$. For one void layer, two void layers and three void layers PDMS ferroelectret, the optimized thickness ratio are achieved around 0.8, 0.6 and 0.55 , respectively. When stacking more void layers together up to 4 layers, it is found that $d_{33}$ of PDMS ferroelectret have a significant improvement. The maximum piezoelectric coefficients $d_{33}$ are achieved by 4-layer PDMS ferroelectret, about 520 $\mathrm{pC} / \mathrm{N}$. When 5 or more layers are stacked, $d_{33}$ does not increase any further. This may be due to an increase in Young's modulus of the ferroelectrets as the number of void layers increases, resulting in a decrease in piezoelectricity.

The energy harvesting performance of fabricated PDMS ferroelectrets are also investigated. The parallelogram void PDMS ferroelectret with the maximum piezoelectric coefficients $520 \mathrm{pC} / \mathrm{N}$ and size of $2 \mathrm{~cm} \times 2 \mathrm{~cm}$ generates maximally $13 \mathrm{~V}, 2.73 \mu \mathrm{W}$ with a $65 \mathrm{M} \Omega$ loading under a compressive force of $800 \mathrm{~N}$ in normal walking model. It is also found that the output voltage increases linearly with increasing forces. The maximum output voltage peak is affected by the step frequency. In other word, the peak voltage is directly proportional to the slope of applied force. In addition, the duration of output is determined by duration of applied force increasing and cannot enhanced by force holding duration increasing. It means that the static force cannot improve the energy output.

In conclusion, a numerical FEA model for predicting the piezoelectricity of the ferroelectret is developed and briefly verified. The piezoelectricity of the fabricated ferroelectret can be tailored by the adjusting the dimensions of the voids and also effected by the shape of void. The demonstrated PDMS ferroelectret can potentially serve as flexible and wearable electromechanical materials, and fulfils the requirements of a variety of wearable sensor and energy harvesting applications.

\section{References}

[1] Hindrichsen C C, Lou-Moller R, Hansen K and Thomsen E V Advantages of PZT thick film for MEMS sensors Sensors Actuators A 163 9-14 (2010)

[2] Park K-I et al. Highly-efficient, flexible piezoelectric PZT thin film nanogenerator on plastic substrates Adv. Mater. 26 2514-20 (2014)

[3] Hillenbrand J and Sessler G M High-sensitivity piezoelectric microphones based on stacked cellular polymer films J. Acoust. Soc. Am. 116 3267-70 (2004)

[4] W. Heywang, K. Lubitz and W. Wersing. Piezoelectricity: evolution and future of a technology, Springer, Berlin Heidelberg, Vol. 114, Ch. 6, pp157 - 177, 2008. 
[5] S. P. Beeby, J. M. Tudor and N. M. White, "Energy harvesting vibration sources for microsystems applications”, Meas. Sci. Technol., Vol. 17, No. 12, pp. R175-R195, 2006.

[6] A. Erturk and D. J. Inman, Piezoelectric energy harvesting, John Wiley \& Sons, 2011.

[7] H. B. Radousky and H. Liang, "Energy harvesting: an integrated view of materials, devices and applications", Nanotechnology, Vol. 23, No. 50, 502001, 2012.

[8] Priya S, Inman DJ. Energy Harvesting Technologies. Berlin: Springer-Verlag Berlin, (2009).

[9] T. J. Kazmierski and S. Beeby. Energy harvesting systems principles, modeling and applications, Springer Science, Berlin, Heidelberg. 2011

[10] Gerhard-Multhaupt R. Less can be more: holes in polymers lead to a new paradigm of piezoelectric materials for electret transducers IEEE Trans. Dielectr. Electr. Insul. 9 850-9 (2002) [11] Bauer S, Gerhard-Multhaupt R and Sessler G M Ferroelectrics: soft electroactive foams for transducers Phys. Today 57 37-43 (2004)

[12] Hu Z and von Seggem H Breakdown-induced polarization buildup in porous fluoropolymer sandwiches: a thermally stable piezoelectret J. Appl. Phys. 99024102 (2006)

[13] J. Raukola, A new technology to manufacture polypropylene foam sheet and biaxially oriented foam films, Dissertation, VTT Publications 361, Technical Research Center of Finland, Tampere University of Technology, (1998)

[14] M. Paajanen, H. Minkkinen, and J. Raukola, Gas diffusion expansion increased thickness and enhanced electromechanical response of cellular polymer electret films, Proceedings, 11th International Symposium on Electrets, 1-3 October 2002, Melbourne, Australia (IEEE Service Center, Piscataway, NJ, USA 2002), pp. 191-194.

[15] M. Wegener, W. Wirges, J. Fohlmeister, B. Tiersch, and R. Gerhard-Multhaupt, Two-step inflation of cellular polypropylene films: void-thickness increase and enhanced electromechanical properties, J. Phys. D: Appl. Phys., vol. 37 (4), pp. 623-627, (2004).

[16] J.-J. Wang, T.-H. Hsu, C.-N. Yeh, J.-W. Tsai, and Y.-C. Su, Piezoelectric polydimethylsiloxane films for MEMS transducers, Micromech Microeng Vol. 22 015013, (2012)

[17] A. Kachroudi, S. Basrour, L. Rufer, A. Sylvestre, and F. Jomni, Micro-structured PDMS piezoelectric enhancement through charging conditions. Smart Materials and Structures, 25(10), p.105027, 2016

[18] Shi J, Zhu D, Cao Z and Beeby S.P. Optimization of a PDMS structure for energy harvesting under compressive forces J. Phys.: Conf. Ser. 66012041 (2015)

[19] Wang J-J, Hsu T-H, Yeh C-N, Tsai J-W and Su Y-C Piezoelectric polydimethylsiloxane films for MEMS transducers J. Micromechanics Microengineering 22015013 (2012)

[20] Wang Y, Li T, Yang H. 2013 Nanofabrication, effects and sensors based on micro-electromechanical systems technology. Phil. Trans. R. Soc. A 371, 20120315. (2012)

[21] Chuah, Y. J., et al. The effects of poly(dimethylsiloxane) surface silanization on the mesenchymal stem cell fate. Biomaterials Science 3(2): 383-390. (2015)

[22] Koschwanez, J. H., Carlson, R. H. \& Meldrum, D. R. Thin PDMS Films Using Long Spin Times or Tert-Butyl Alcohol as a Solvent. PLoS ONE 4, e4572 (2009).

[23] Zhang, W. Y., Ferguson, G. S. \& Tatic-Lucic, S. Elastomer-supported cold welding for room temperature wafer-level bonding. in Micro Electro Mechanical Systems, 17th IEEE International Conference on. (MEMS) 741-744 (2004).

[24] J. E. A. Bertram, Constrained optimization in human walking: cost minimization and gait plasticity, Journal of experimental biology, Vol. 208, No. 6, pp. 979-991, 2005.

[25] J. E. A. Bertram and A. Ruina, Multiple walking speed-frequency relations are predicted by constrained optimization, Journal of theoretical Biology, Vol. 209, No. 4 pp. 445-453, 2001.

[26] G. A. Cavagna and P. Franzetti, The determinants of the step frequency in walking in humans, The Journal of Physiology, Vol. 373, No. 1, pp. 235-242, 1986

[27] Y. Rao, K. M. McEachern and D. P. Arnold, A compact human-powered energy harvesting system, In Journal of Physics: Conference Series, Vol. 476, No. 1, pp. 012011, IOP Publishing, 2013. 
[28] A. P. Chandrakasan, S. Sheng and R. W. Brodersen, Low-power CMOS digital design, IEICE Transactions on Electronics, Vol. 75, No. 4, pp. 371-382, 1992

[29] Döring J, Bovtun V, Gaal M, Bartusch J, Erhard A, Kreutzbruck M and Yakymenko Y Piezoelectric and electrostrictive effects in ferroelectret ultrasonic transducers J. Appl. Phys. 112 084505 (2012)

[30] Zhang X, Huang J, Wang X and Xia Z Piezoelectricity and dynamic characteristics of laminated fluorocarbon films IEEE Trans. Dielectr. Electr. Insul. 17 1001-7 (2010)

[31] V. Kursun, S. G. Narendra, V. K. De and E. G. Friedman, Low-voltage-swing monolithic dcdc conversion, IEEE Transactions on Circuits and Systems II: Express Briefs, Vol. 51, No. 5, pp. 241-248, 2004.

[32] G. Yang, G, B .H. Stark, S. J. Hollis, S. G. Burrow, Challenges for energy harvesting systems under intermittent excitation, Emerging and Selected Topics in Circuits and Systems, IEEE Journal on, doi:10.1109/JETCAS.2014.2337172.

[33] Luo, Z, Zhu, D, Shi, J, Beeby, S, Zhang, C, Proynov, P \& Stark, B, Energy harvesting study on single and multilayer ferroelectret foams under compressive force IEEE Transactions on Dielectrics and Electrical Insulation, vol. 22, no. 3, 7116323, pp. 1360-1368. (2015) 